\title{
Protein microarray technology and ultraviolet crosslinking combined with mass spectrometry for the analysis of protein-DNA interactions
}

\author{
Birgit Kersten,* Alexandra Possling, Franca Blaesing, Ekaterina Mirgorodskaya, \\ Johan Gobom, and Harald Seitz
}

Department Lehrach, Max Planck Institute for Molecular Genetics, Ihnestrasse 73, D-14195 Berlin, Germany

Received 20 February 2004

Available online 17 June 2004

\begin{abstract}
To gain insights into complex biological processes, such as transcription and replication, the analysis of protein-DNA interactions and the determination of their sequence requirements are of central importance. In this study, we probed protein microarray technology and ultraviolet crosslinking combined with mass spectrometry (MS) for their practicability to study protein-DNA interactions. We chose as a model system the well-characterized interaction of bacterial replication initiator DnaA with its cognate binding site, the DnaA box. Interactions of DnaA domain 4 with a high-affinity DnaA box (R4) and with a low-affinity DnaA box (R3) were compared. A mutant DnaA domain 4, A440V, was included in the study. DnaA domain 4, wt, spotted onto FAST slides, revealed a strong signal only with a Cy5-labeled, double-stranded, 21-mer oligonucleotide containing DnaA box R4. No signals were obtained when applying the mutant protein. Ultraviolet crosslinking combined with nanoLC/MALDI-TOF MS located the site of interaction to a peptide spanning amino acids 433- 442 of Escherichia coli DnaA. This fragment contains six residues that were identified as being involved in DNA binding by recently published crystal structure and nuclear magnetic resonance (NMR) analysis. In the future, the technologies applied in this study will become important tools for studying protein-DNA interactions.
\end{abstract}

(C) 2004 Elsevier Inc. All rights reserved.

Keywords: Protein microarray technology; Fluorescence detection; NanoLC-MALDI-TOF MS; DnaA domain 4; DnaA box

Protein-DNA interactions drive basic cellular processes such as transcription, replication, and recombination. The identification of such interactions and the determination of their specificity by measuring the effects of mutations on the interaction are important to understand these processes.

Direct methods, such as in vivo footprinting [1], allow target identification of single DNA-binding proteins. A genome-wide location of DNA-binding proteins is enabled by the ChIP-chip approach, which combines chromatin immunoprecipitation with cDNA microarray hybridization and has recently been used to map transcriptional networks in yeast [2]. However, this method is limited by the need for antibodies against the

\footnotetext{
${ }^{*}$ Corresponding author. Fax: +49-30-8413 1128.

E-mail address: birgit.kersten-berlin@gmx.de (B. Kersten).
}

DNA-binding proteins analyzed and does not easily allow including mutant DNA-binding proteins in the analysis.

Several in vitro methods have been used for the analysis of protein-DNA interactions. For a given DNAbinding protein, in vitro selection [3] has permitted the sampling of multiple DNA-binding sites. However, this method provides only a partial view of the binding site specificity because only the strongest interactions are selected. This limitation has been overcome by the development of dsDNA arrays for studying the DNA sequence specificity of protein-DNA interactions [4].

For given target DNA sites, phage display has emerged as a powerful tool, for example, for selecting transcription factors that recognize the given target out of millions of protein variants [5]. To study the effects of individual protein mutants on the interaction, other methods that permit analysis of only one interaction at a 
time, such as gel mobility shift analysis [6], Southwestern blotting [5], enzyme-linked immunosorbent assay (ELISA) ${ }^{1}$-based methods [7], and Biacore analysis [6,7], had to be used. However, these methods are costly and are limited by low throughput. These limitations may be overcome by protein array-based methods, which allow the analysis of hundreds or even thousands of proteins (mutated or nonmutated) at once against a molecular target in a highly parallel fashion [8,9]. Initial attempts to apply this technology to the analysis of protein-DNA interactions were made by Ge [10] using macroarrays generated by dot blotting. Recently, membrane arrays combined with near-infrared fluorescence detection have been used to characterize protein-DNA interactions [11].

In the current proof-of-principle study, we demonstrate the power of the protein microarray technology to screen for protein-DNA interactions. We also show that complementary information can be obtained by combining this technique with nanoLC/MALDI-TOF mass spectrometry (MS) analysis of ultraviolet (uv)-crosslinked interactants, allowing one to determine the part of the protein involved in the interaction.

We analysed the protein DnaA from Escherichia coli as a well-characterized DNA-binding protein. DnaA is the key protein for the initiation of bacterial replication from the chromosomal origin, oriC, of E. coli and other bacteria [12]. The DnaA protein recognizes and binds specifically to consensus sequences in oriC, termed DnaA boxes (R1-R4 and M) [13]. In addition to its central role in the initiation of replication, DnaA acts as a transcription factor of various genes [14]. On binding to DnaA box motifs located within promoters or coding regions of these genes, DnaA can act as a repressor, an activator, or a terminator of transcription [15].

DnaA binds to a nonpalindromic 9-bp consensus sequence $5^{\prime}$-T-T-A/T-T-N-C-A-C-A or a close match to it $[13,16,17]$. The binding efficiency of DnaA is affected by this 9-bp consensus sequence and by the flanking sequences [18] and involves both strands [19]. Dissociation constants for binding of DnaA to individual DnaA boxes vary between $1 \mathrm{nM}$ (DnaA boxes R1 and R4) and more than $200 \mathrm{nM}$ (unspecific binding, DnaA box R3) [18]. Based on sequence alignments, the DnaA protein can be subdivided into four domains [20] with distinct biochemical functions [21]. The C-terminal domain, domain 4 (aa 374-467), of E. coli DnaA is necessary and sufficient for specific DNA binding in vitro and in vivo [6,22]. Several amino acid residues were identified as being involved in DNA binding by an extensive characterization of the DNA-binding domain using mutants of DnaA [6]. To study the reli-

\footnotetext{
${ }^{1}$ Abbreviations used: ELISA, enzyme-linked immunosorbent assay; MS, mass spectrometry; uv, ultraviolet; dsOligos, double-stranded oligonucleotides; TBST, TBS/0.1\% (v/v) Tween 20; $\mathrm{m} / \mathrm{z}$, mass-to-charge ratio; NMR, nuclear magnetic resonance.
}

ability of the protein microarray assay, we included one of the nonbinding mutants, the A440V DnaA [6], in this study.

\section{Materials and methods}

\section{Oligonucleotides}

All oligonucleotides used in this study for cloning, gel retardation, protein microarray experiments, and crosslinking are listed in Table 1. Unless stated otherwise, complementary oligonucleotides were annealed to obtain double-stranded oligonucleotides (dsOligos) by mixing $10 \mu \mathrm{l}$ of the appropriate oligonucleotides $(100 \mathrm{pmol} / \mu \mathrm{l})$ with $10 \mu 110 \times$ Tris-acetate buffer $(330 \mathrm{mM}$ Tris, $\mathrm{pH}$ 7.6; $660 \mathrm{mM} \mathrm{Mg}$-acetate), heating to $95^{\circ} \mathrm{C}$ and subsequently cooling to room temperature, and adjusting to $1 \times$ Trisacetate buffer afterwards.

\section{Construction of expression vector $p Q E 30 p s$}

Oligonucleotides pQE30_PS forw. and pQE30_PS rev. (Table 1) were hybridized and ligated into the BamHIopened pQE30 (Qiagen, Hilden, Germany). The resulting vector clone $\mathrm{pQE} 30 \mathrm{ps}$ contains a site for the precision protease to remove the RGS-His ${ }_{6}$ tag after purifying the tagged proteins.

Table 1

Oligonucleotides for cloning, gel retardation, protein microarray, and crosslinking experiments

\begin{tabular}{|c|c|}
\hline Name & Sequence \\
\hline \multicolumn{2}{|l|}{ Cloning } \\
\hline pQE30_PS forw. & $\begin{array}{l}\text { 5'-GATCTCTGGAAGTTCTTCTTCC } \\
\text { AGGGGCCCCTGG }\end{array}$ \\
\hline pQE30_PS rev. & $\begin{array}{l}\text { 5'-GATCCCAGGGGCCCCTGGA } \\
\text { ACAGAACTTCCAGA }\end{array}$ \\
\hline DnaA domain IV forw. & $\begin{array}{l}\text { 5'-GGCGGATCCGTCACCATCGAC } \\
\text { AATATTCAGAAGAC }\end{array}$ \\
\hline DnaA domain IV rev. & $\begin{array}{l}\text { 5'-GGCAAGCTTTTACGATGACAA } \\
\text { TGTTCTGATTAAATTTG }\end{array}$ \\
\hline \multicolumn{2}{|l|}{ Gel retardation } \\
\hline Box $\mathrm{R} 4 / o r i C$ upper & 5'-ACAGAGTTATCCACAGTAGAT \\
\hline Box $\mathrm{R} 4 /$ oriC lower & 5'-ATCTACTGTGGATAACTCTGT \\
\hline Nonbinding upper & 5'-ATCAGTCACGTGATCAGATCA \\
\hline Nonbinding lower & 5'-TGATCTGATCACGTGACTGAT \\
\hline \multicolumn{2}{|l|}{ Microarray experiments } \\
\hline Box $\mathrm{R} 4 /$ oriC upper & 5'-ACAGAGTTATCCACAGTAGAT \\
\hline $\begin{array}{l}\text { Box } \mathrm{R} 4 / \text { oriC-Cy } 5 \\
\text { lower }\end{array}$ & $\begin{array}{l}\text { 5'-Cy5-ATCTACTGTGGATAACTCT } \\
\text { GT }\end{array}$ \\
\hline Box $\mathrm{R} 3 /$ oriC upper & 5'-CGGTAGTTATCCAAAGAACAA \\
\hline $\begin{array}{l}\text { Box } \mathrm{R} 3 / \text { oriC-Cy5 } \\
\text { lower }\end{array}$ & $\begin{array}{l}\text { 5'-Cy5-TTGTTCTTTGGATAACTAC } \\
\text { CG }\end{array}$ \\
\hline \multicolumn{2}{|l|}{ Crosslinking } \\
\hline $\begin{array}{l}\text { Box R4/oriC-BrdU } \\
\text { upper }\end{array}$ & $\begin{array}{l}\text { 5'-XACAGAGTTAUCCACAGTAG } \\
\text { AT }\end{array}$ \\
\hline Box $\mathrm{R} 4 /$ oriC lower & 5'-ATCTACTGTGGATAACTCTGT \\
\hline
\end{tabular}

Note. X, fluorescein label; $\mathrm{U}$, bromdeoxyuracil. Bold letters indicate the DnaA box. 


\section{Cloning of wt DnaA domain 4 and A440V DnaA domain 4}

wt DnaA domain 4 (aa 374-467) was amplified with oligonucleotides DnaA domain IV forw. and DnaA domain IV rev. (Table 1) using pdnaA116 [6] as template. The PCR product was restricted with BamHI/HindIII and ligated with pQE30ps previously digested with the same restriction enzymes.

In the case of A440V DnaA domain 4 (aa 374-467), the DNA was amplified with the same oligonucleotides as was the $w t$ protein using pDnaA116A440V [6] as template. All clones were verified by DNA sequencing.

\section{Control clone}

As the control clone, we used a cDNA expression clone from a human fetal brain library [23] expressing protein Drebrin F (Swiss-Prot accession: Q9UJU6) as an N-terminal fusion protein with an $\mathrm{RGS}-\mathrm{His}_{6}$ tag.

\section{Expression and purification of the proteins}

Expression of His-tagged DnaA domain 4, A440V DnaA domain 4, and control protein was performed in E. coli WM1704 cells (araD139, chr::Tn10, del(lacU169 proA+), del(lon), hflA150, rpsL) [24] carrying the plasmid pSE111 with the 1 acI $\mathrm{I}^{\mathrm{Q}}$ repressor and the $\arg U$ gene for a rare arginine tRNA [25]. For expression, the cells were induced at an $\mathrm{OD}_{600}$ of 0.6 with $1 \mathrm{mM}$ IPTG for $60 \mathrm{~min}$ at $37^{\circ} \mathrm{C}$. Cells harvested from $1 \mathrm{~L}$ induced culture were resuspended in $10 \mathrm{ml}$ lysis buffer $(40 \mathrm{mM}$ Hepes, $\mathrm{pH}$ 7.6; $700 \mathrm{mM} \mathrm{KCl} ; 50 \mathrm{mM}$ Mg-acetate; $10 \mathrm{mM}$ imidazol; $5 \%$ glycerol; $5 \mathrm{mM} \quad \beta$-mercaptoethanol; $0.02 \% \mathrm{NaN}_{3}$ ), sonificated, and centrifugated. The cleared lysate was loaded onto an equilibrated NiNTA column (Qiagen). His-tagged proteins were eluted with G-100 buffer ( $40 \mathrm{mM}$ Hepes, $\mathrm{pH} 7.6 ; 100 \mathrm{mM}$ K-glutamate; $10 \mathrm{mM}$ Mg-acetate; $5 \%$ glycerol; $5 \mathrm{mM} \beta$-mercaptoethanol; $0.02 \% \mathrm{NaN}_{3}$ ) plus $250 \mathrm{mM}$ imidazol.

In the cases of $w t$ and A440V DnaA domain 4, the eluates were subsequently loaded onto a cation exchange column (SP-Sepharose, Amersham Biosciences, Buckinghamshire, UK). Proteins were eluted with a 150 to $1000 \mathrm{mM}$ K-glutamate gradient in G-100 buffer. Protein containing peak fractions were determined by SDS-PAGE, pooled, and concentrated with an Amicon Ultra 10 device (Millipore, Bedford, MA, USA) to $5 \mathrm{mg} / \mathrm{ml}$ (DnaA domain 4) or $1 \mathrm{mg} / \mathrm{ml}$ (A440V DnaA domain 4) and were stored at $-80^{\circ} \mathrm{C}$. Fulllength DnaA (aa 1-467) was expressed and purified as described previously [6].

\section{Gel retardation assay}

Binding of full-length DnaA protein (aa 1-467), DnaA domain 4 (aa 374-467), and A440V DnaA domain 4 to dsOligo substrates was analyzed by band shift assays on $2 \%$ agarose gels (Seakem LE, FMC Bioproducts, Rockland, ME, USA). Proteins were incubated with a 21-mer dsOligo containing DnaA box R4 and with a nonbinding 21-mer dsOligo (Table 1). Protein aliquots were thawed and diluted with binding buffer (20 mM Hepes/KOH, pH 8.0; $5 \mathrm{mM}$ Mg-acetate; $1 \mathrm{mM}$ EDTA; $4 \mathrm{mM}$ DTT) to a final concentration of $2 \mathrm{pmol} / \mu \mathrm{l}$ on ice. Assay mixtures $(16 \mu l)$ were assembled by mixing the desired protein $(4 \mathrm{pmol})$ with 6 pmol DNA substrate in $1 \times$ binding buffer on ice. Binding of the proteins to DNA was achieved by incubation of the reaction for $5 \mathrm{~min}$ on ice and an additional $10 \mathrm{~min}$ at $30^{\circ} \mathrm{C}$. The samples were placed on ice until loading to the gel. Electrophoresis was carried out at room temperature for $90 \mathrm{~min}$ at $4 \mathrm{~V} \mathrm{~cm}^{-1}$ in $0.5 \times \mathrm{TBE}$ buffer $(44.5 \mathrm{mM}$ Tris-borate; $1.0 \mathrm{mM}$ EDTA, pH 8.0). Gels were stained with VISTRA-Green (Molecular Probes Europe, Leiden, Netherlands) in electrophoresis buffer according to the manufacturer's instructions. Stained gels were read with a FluorImager 575, and images were further processed with ImageQuant NT5.1 software (Molecular Dynamics, Krefeld, Germany).

\section{Generation of protein microarrays}

FAST slides (Schleicher and Schuell, Dassel, Germany) were placed in a Q-array system (Genetix, New Milton, UK) equipped with humidity control (65\%) and 16 blunt-end stainless-steel pins with a tip diameter of $150 \mu \mathrm{m}$.

DnaA domain 4, A440V DnaA domain 4, and the control protein $(500 \mu \mathrm{g} / \mathrm{ml}$ each) were spotted. BSA $(500 \mu \mathrm{g} / \mathrm{ml})$ and protein elution buffers were used as controls. All samples were spotted using a $4 \times 4$ spotting pattern (test spot) with 16 spots per sample. Each spot was loaded once, transferring a volume of $0.6 \mathrm{nl}$ per spot (dot pitch 950 microns and spotting depth 150 microns).

\section{Screening of FAST slides with anti-RGS-His antibody $_{6}$}

The slides were blocked for $1 \mathrm{~h}$ at room temperature using 2\% BSA/TBST (TBS/0.1\% (v/v) Tween 20). Mouse-anti-RGS-His ${ }_{6}$ antibody (Qiagen) was diluted in blocking solution (1:2000) and then applied onto the arrays for $1 \mathrm{~h}$ at room temperature, followed by two 10min wash steps with TBST. The slides were further incubated for $1 \mathrm{~h}$ at room temperature with a Cy3-labeled secondary antibody (rabbit anti-mouse immunoglobulin G) (Dianova, Hamburg, Germany), which was applied using a 1:800 dilution in blocking solution. Then two wash steps of $30 \mathrm{~min}$ each and one wash step of $60 \mathrm{~min}$ were performed in TBST. All antibody incubation steps were carried out in a $200-\mu 1$ volume underneath a cover slide and in the dark. 
Screening of FAST slides with dsOligos

Complementary oligonucleotides for DnaA box R4 and DnaA box R3 (Table 1) were mixed (each $10 \mu 1$ of a $100-\mathrm{pmol} / \mu \mathrm{l}$ solution) and were annealed by boiling for $5 \mathrm{~min}$ and slow cooling to room temperature (protected from light). The Cy5-labeled dsOligos (R4/oriC and R3/ oriC) were diluted with aqua bidest to a stock concentration of $10 \mathrm{pmol} / \mu \mathrm{l}$ and stored at $-20^{\circ} \mathrm{C}$.

To screen on FAST slides, dsOligo dilutions were prepared with $2 \%$ BSA/TBST and the addition of $48 \mu \mathrm{M}$ poly-d[IC]. First, the slides were blocked for $1 \mathrm{~h}$ at room temperature with $300 \mu 12 \%$ BSA/TBST. Then the slides were incubated with the dsOligo dilutions $(100 \mu \mathrm{l})$ for $1 \mathrm{~h}$ at room temperature and then washed in two wash steps: one for $30 \mathrm{~min}$ and another for $45 \mathrm{~min}$ with TBST. All incubation steps were carried out underneath a cover slide and in the dark. The signal detection was performed with the aid of a ScanArray 4000 device (PerkinElmer, Cologne, Germany).

\section{Image analysis}

The median spot intensity data (background subtracted) were obtained with the GenePixPro 4.0 software. For further comparison, the average values of 16 spots have been calculated.

\section{uv crosslinking of DnaA domain 4 with DnaA box $R 4$}

uv crosslinking of His-tagged DnaA (374 467) was performed with a 21-mer dsOligo containing DnaA box R4 that was generated by annealing the oligonucleotides Box $\mathrm{R} 4 /$ oriC-BrdU upper and Box R4/oriC lower (Table 1).

\section{Analytical scale}

Protein $(50 \mathrm{pmol})$ was incubated with DNA in various molar ratios (5:1, 2:1, 1:1, 1:2, and 1:5). All reactions were done in microtiter plates in a final volume of $20 \mu \mathrm{l}$ in modified Marians buffer $(20 \mathrm{mM}$ Hepes, $5 \mathrm{mM}$ Mgacetate, $1 \mathrm{mM}$ EDTA, $4 \mathrm{mM}$ DTT, and $100 \mu \mathrm{M}$ ATP). Samples were incubated for $15 \mathrm{~min}$ at $30^{\circ} \mathrm{C}$, placed on ice, and irradiated with uv light $(256 \mathrm{~nm})$ for various times (5-60 min) in a uv crosslinker (Stratagene, La Jolla, CA, USA) at a distance of $5 \mathrm{~cm}$ from the light source. Samples were then mixed with one-tenth volume of $10 \times$ Lugtenberg buffer $(625 \mathrm{mM}$ Tris- $\mathrm{HCl}, \mathrm{pH} 6.8$; $10 \%$ mercaptoethanol; $20 \%$ SDS; $0.2 \%$ Bromphenol blue), and crosslinked protein was separated from noncrosslinked material by SDS-PAGE. Reaction products were visualized using a FluorImager (Amersham Biosciences) for fluorescein-labeled oligonucleotides and with Coomassie staining for the protein moiety. Optimal results were obtained with protein and DNA in a molar ratio of $1: 1$ and after $15 \mathrm{~min}$ of irradiation.

\section{Preparative scale}

For the preparative scale, multiple samples of 200 pmol protein and 200 pmol dsOligo were incubated and irradiated as described for the analytical scale reactions. Crosslinked DnaA (aa 374-467) was isolated by preparative SDS-PAGE. The band of interest was excised from the gel and stored at $-20^{\circ} \mathrm{C}$ until further processing. Likewise, the noncrosslinked protein band was cut out and kept as a reference sample.

\section{Mass spectrometric analysis of DnaA domain 4}

Excised gel samples were washed three times for $30 \mathrm{~min}$ in $200 \mu \mathrm{l} 50 \%$ acetonitrile (v/v). Water was then expelled from the gel piece by incubation with $200 \mu 1$ acetonitrile for $5 \mathrm{~min}$, followed by lyophilization in a vacuum centrifuge for $10 \mathrm{~min}$. An 8- $\mu 1$ aliquot of trypsin solution $\left(12 \mathrm{ng} / \mu \mathrm{l} ; 50 \mathrm{mM} \mathrm{NH}_{4} \mathrm{HCO}_{3}, \mathrm{pH} 7.8\right)$ was added to the gel samples. After absorption of the trypsin solution, digestion buffer $\left(50 \mathrm{mM} \mathrm{NH} \mathrm{m}_{4} \mathrm{HCO}_{3}\right)$ was added to cover the gel samples. Digestion was performed at $37^{\circ} \mathrm{C}$ overnight.

MALDI-TOF MS was performed on a Reflex III instrument (Bruker Daltonics, Germany). CHCA was used as the MALDI matrix, and the samples were prepared as described previously [26]. MS/MS data were acquired on an Ultraflex instrument equipped with LIFT technology (Bruker Daltonics). Data were analyzed using the FlexAnalysis software provided by the instrument manufacturer.

An in-house-developed offline nanoLC-MALDI interface was used for fractionation of nanoLC effluent at a flow of $200 \mathrm{nl} / \mathrm{min}$ (UltiMate, LC Packings, Netherlands) directly onto prepared MALDI matrix layers [27]. A reverse-phase capillary column $(15 \mathrm{~cm}, 75 \mu \mathrm{m}$ i.d., PepMap column, LC Packings) was used for separation of the in-gel tryptic digests using a binary gradient of buffer A ( $1 \%$ acetonitrile $(\mathrm{v} / \mathrm{v}), 0.05 \%$ trifluoroacetic acid $(\mathrm{v} / \mathrm{v}))$ and buffer B (90\% acetonitrile (v/v), 0.04\% trifluoroacetic acid $(\mathrm{v} / \mathrm{v}))$ at $1.5 \%$ buffer $\mathrm{B} / \mathrm{min}$. Initiation and termination of fraction collection were triggered from the LC system at time points defined within the separation gradient. For each sample, 70 fractions, each corresponding to $20 \mathrm{~s}$, were collected onto the MALDI sample support. Detection of compounds in the deposited LC fractions was performed using a Reflex III MALDITOF instrument.

Mass spectrometric data processing was performed using in-house-developed software that performs spectra calibration according to a method described previously [28], followed by filtering of background signals. Further processing of LC-MALDI MS data involved grouping of the signals detected into different fractions according to mass-to-charge ration $(\mathrm{m} / \mathrm{z})$ and LC elution time, resulting in a peptide profile in which each 
peptide is characterized by its detected mass and elution time.

\section{Results}

\section{Results of gel retardation experiment}

His-tagged proteins were expressed, purified, and analyzed using SDS-PAGE (data not shown). Prior to the uv crosslinking and microarray experiments, DnaA domain 4 and A440V DnaA domain 4 (aa 374-467) were tested for their binding properties to DNA by a gel retardation assay in comparison with the full-length DnaA protein (aa 1-467). A synthetic 21-mer dsOligo containing the DnaA box R4 and a nonbinding dsOligo were used [6] (Table 1). The binding reaction was carried out as described. Complex formation between $w t$ DnaA domain 4 and DnaA box R4 is in the same concentration range as with full-length DnaA. The full-length DnaA has a molecular weight of approximately $52 \mathrm{kDa}$ compared with approximately $13 \mathrm{kDa}$ for $w t$ DnaA domain 4 . The higher molecular mass results in a more retained band in the gel retardation experiment (lanes 3 and 5 in Fig. 1). The mutant A440V DnaA domain 4 was unable to bind specifically to DnaA box R4 (lane 7 in Fig. 1). No binding at all was detectable to the nonbinding dsOligo (lanes 4, 6, and 8 in Fig. 1).

\section{Protein-DNA interactions on protein microarrays}

We used the interaction of DnaA domain 4 with DnaA box R4, which has been well characterized by the gel retardation experiments, as a model to probe the protein microarray technology for its practicability to study

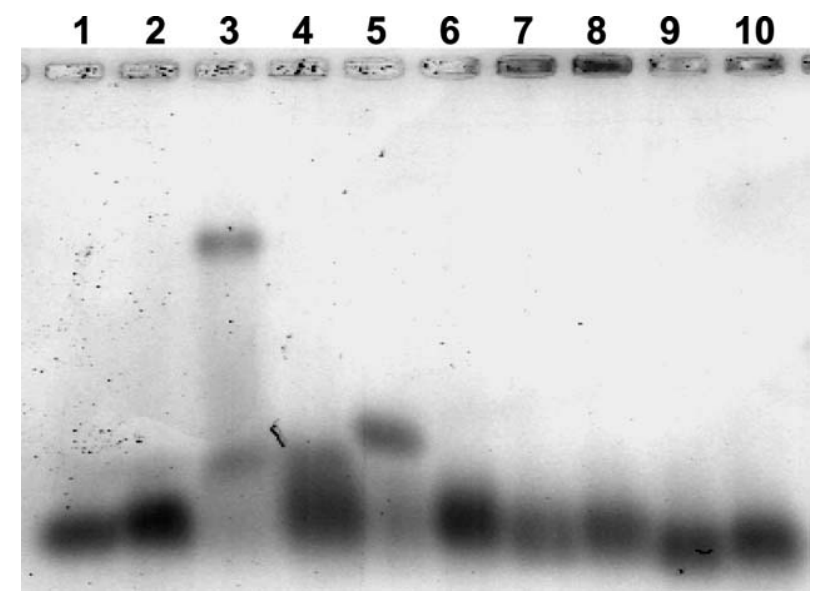

Fig. 1. Gel retardation experiment. DnaA (aa 1-467), DnaA domain 4 (aa 374-467), and A440V DnaA domain 4 were incubated with DnaA box R4 (lanes 3, 5, and 7, respectively) and with a nonbinding dsOligo (lanes 4, 6, and 8, respectively). As controls, the used dsOligos without protein were loaded: DnaA box R4 (lanes 1 and 9) and nonbinding dsOligo (lanes 2 and 10).
protein-DNA interactions. For this purpose, the same dsOligo-containing DnaA box R4 was used as in the gel retardation experiment, but with Cy5 label (Table 1). Protein arrays were generated spotting His-tagged DnaA domain 4, the A440V DnaA domain 4, and a Histagged control protein together with buffer controls onto FAST slides. To detect the immobilized His-tagged proteins, a microarray was incubated with an anti-RGS-His 6 antibody followed by a Cy3-labeled secondary antibody (Fig. 2). As expected, all three His-tagged proteins gave a fluorescence signal, in contrast to the buffers and the BSA control. Incubation of slides spotted in the same series with the $\mathrm{R} 4$ dsOligo resulted in a specific detection of $w t$ DnaA domain 4 on the microarray (sample 1 in Fig. 2). The A440V DnaA domain 4, as well as the His-tagged control protein and the other controls, gave no signals.

To compare the concentration-dependent binding properties of DnaA boxes R4 and R3 to immobilized DnaA domain 4 and its mutant A440V, three series of protein microarrays were spotted as described above. Each array series was incubated in a single experiment with freshly prepared dilution series of Cy5-labeled dsOligos (2-2000 nM) containing the respective DnaA box. Fig. 3A shows the images following incubation of the protein microarrays with $500 \mathrm{nM}$ dsOligo concentration (DnaA box $\mathrm{R} 4 /$ oriC and box $\mathrm{R} 3 /$ oriC, respectively).

For every dsOligo concentration, the mean values of the relative intensities of the 16 spots per sample (DnaA domain 4, A440V DnaA domain 4, and control protein) were determined from the images and plotted against the respective dsOligo concentration as shown in Fig. 3B for one representative experiment out of the three experiments. In the case of DnaA box R4, the mean relative intensity for its binding to DnaA increased with rising dsOligo concentration. The A440V mutant and the control protein gave no specific signals with R4. Only at the highest oligonucleotide concentration was an unspecific binding of $\mathrm{R} 4$ to these proteins detectable. The concentration series with DnaA box R3 revealed only at the highest dsOligo concentrations unspecific signals for DnaA, A440V, and control (Fig. 3B).

uv crosslinking of the wt DnaA domain 4 with DNA and mass spectrometric determination of the interaction site

His-tagged DnaA domain 4 was crosslinked with a dsOligo of DnaA box R4 covering the same DNA sequence as the dsOligos used for the gel retardation and microarray experiments (Table 1). To obtain optimal yields of crosslinked protein, the crosslinking procedure was optimized with respect to the molar ratios of protein and DNA and to the irradiation time as described in Materials and methods. The crosslinked protein was further investigated to locate the region of the protein sequence interacting with DNA. Both crosslinked and noncrosslinked DnaA domain 4 were separated by 


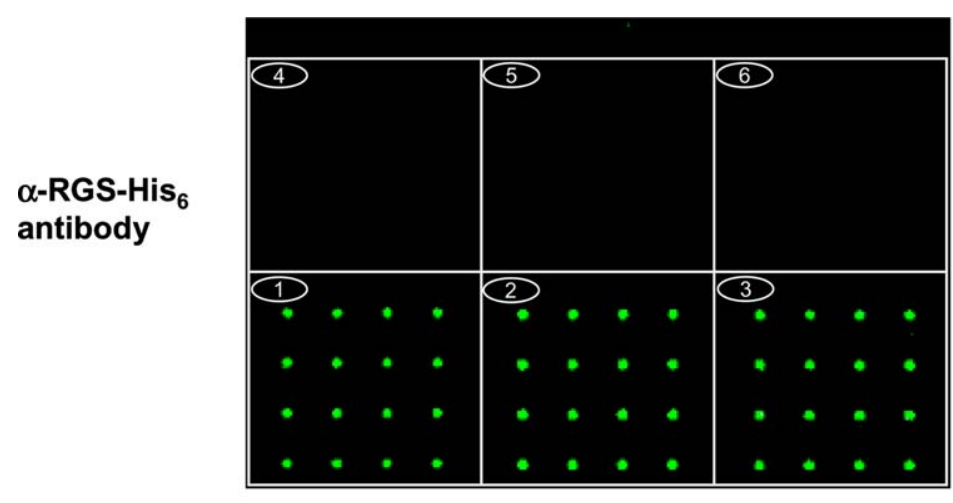

\section{dsOligo of DnaA box R4}

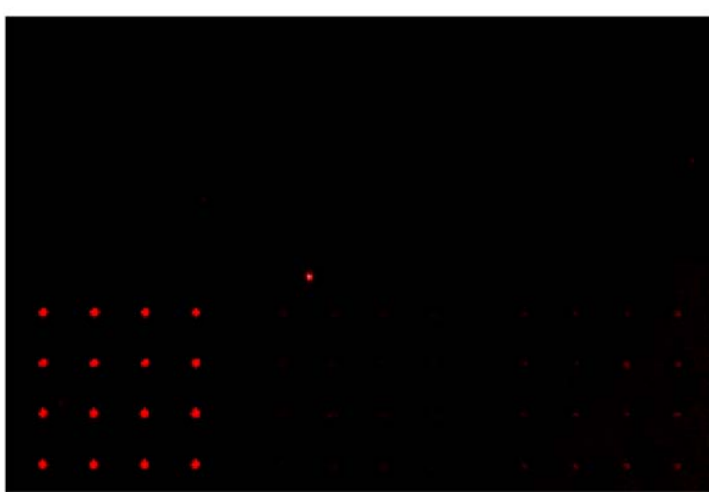

Fig. 2. Fluorescence detection of immobilized His-tagged proteins (array above) and of DNA-protein interaction (array below) on protein microarrays. The purified His-tagged proteins (1-3) and controls (4-6) were spotted on FAST slides using a $4 \times 4$ spotting pattern (test spot) with 16 spots per sample. Each spot was loaded once, transferring a volume of $0.6 \mathrm{nl}$ per spot (dot pitch 950 microns and spotting depth 150 microns). The chips were screened with an anti-RGS-His ${ }_{6}$ antibody, followed by a Cy3-labeled secondary antibody (top image) or the Cy5-labeled dsOligo BoxR4/oriC, $500 \mathrm{pmol} / \mathrm{ml}$ (bottom image): (1) wt DnaA domain 4,500 $\mu \mathrm{g} / \mathrm{ml}$ in G100 buffer; (2) A440V mutant DnaA domain 4,500 $\mu \mathrm{g} / \mathrm{ml}$ in G100 buffer; (3) control protein, $500 \mu \mathrm{g} / \mathrm{ml}$ in G100-buffer plus $250 \mathrm{mM}$ imidazole; (4) G100 buffer; (5) BSA, $500 \mu \mathrm{g} / \mathrm{ml}$ in aqua bidest; (6) G100 buffer plus $250 \mathrm{mM}$ imidazole.
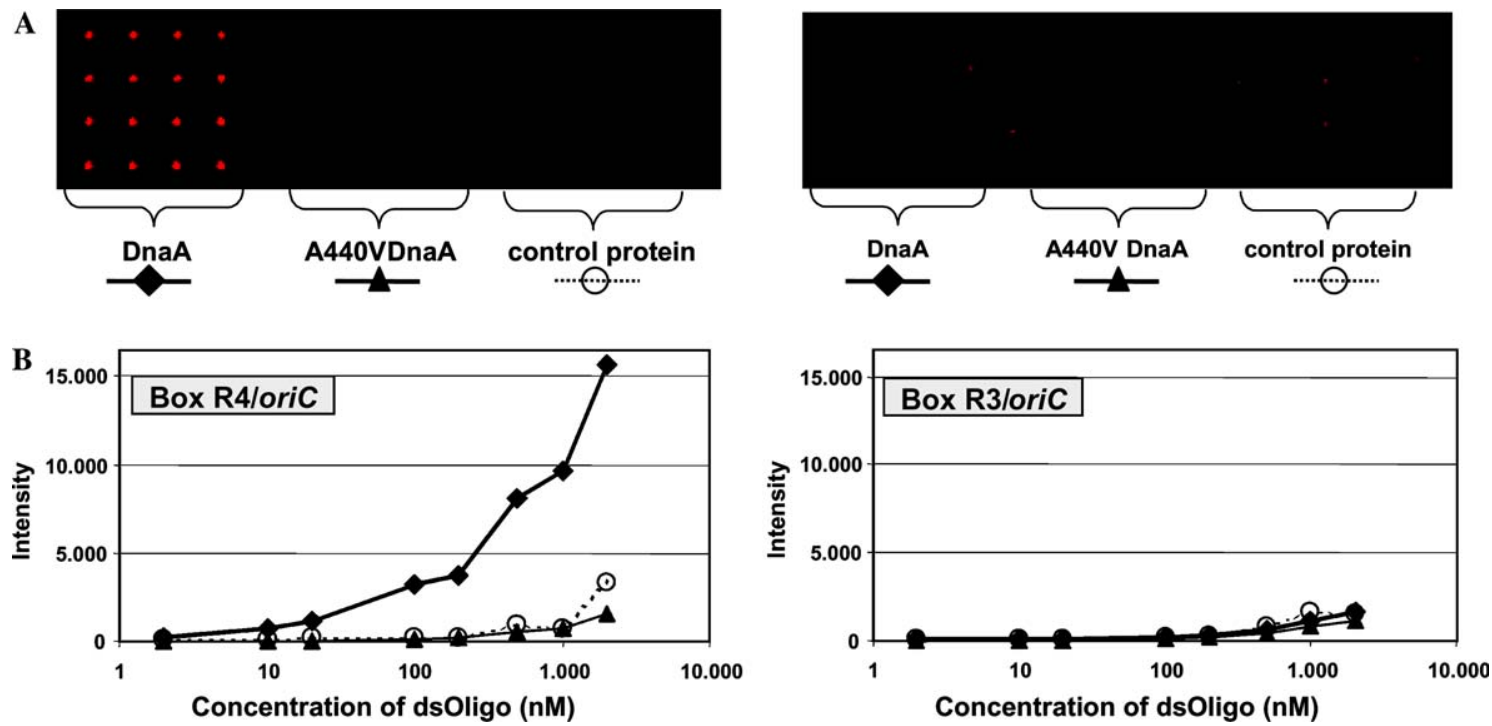

Fig. 3. Concentration-dependent binding of different DnaA boxes to $w t$ and A440V DnaA domain 4 immobilized on protein microarrays. (A) Images obtained following incubation of the protein microarrays with $500 \mathrm{nM}$ of Cy5-labeled DnaA box R4/oriC (left) and box R3/oriC dsOligo (right). (B) Average relative intensities of the fluorescence signals calculated from the protein microarray images, for example, in (A). The purified His-tagged proteins were spotted on FAST slides as described in the Fig. 2. The chips spotted in one series were screened with different concentrations of dsOligos BoxR4/oriC (left) or BoxR3/oriC (right). Mean values of the relative intensities of the 16 spots per sample obtained from one representative experiment were plotted against dsOligo concentrations in (B). 
Protein control

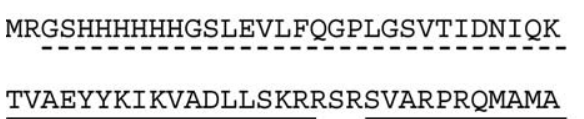

LAKELTNHSLPEIGDAFGGRDHTTVLHACRK
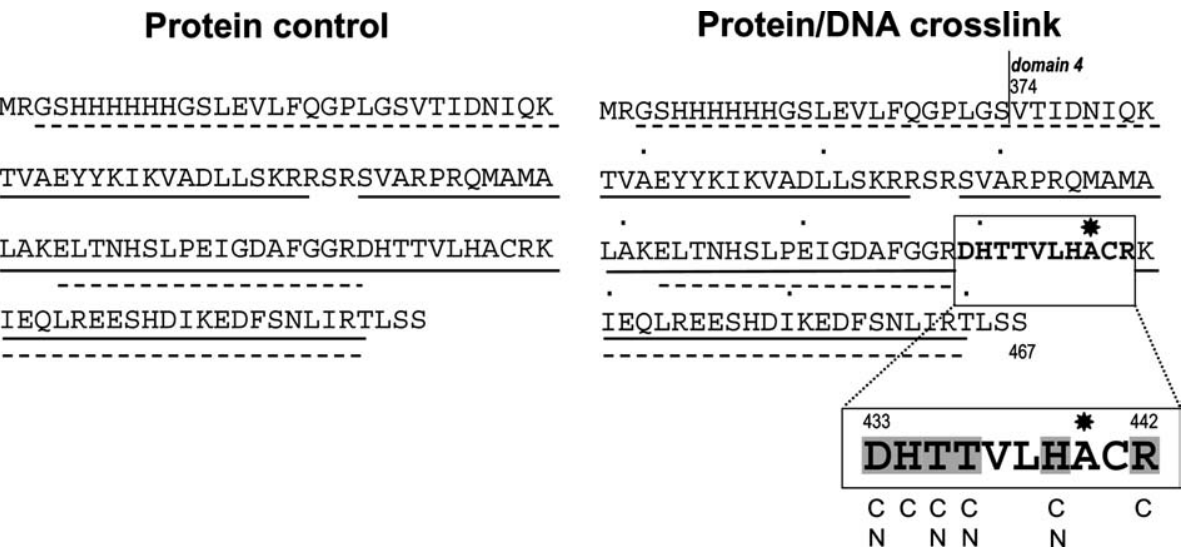

Fig. 4. Protein sequence coverage based on the data obtained by MALDI-TOF MS mixture analysis (dashed line) and nanoLC/MALDI-TOF MS analysis (solid line) of DnaA domain 4 (protein control) and DnaA domain 4 crosslinked with dsOligo BoxR4/oriC (protein-DNA crosslink). Part of the sequence, shown in bold, was identified as tryptic peptide involved in DNA crosslink based on the nanoLC/MALDI-TOF MS. The asterisk (*) marks amino acid $\mathrm{A}^{440}$, which was mutated to $\mathrm{V}$ in domain 4 of the A440V DnaA protein. Gray fields mark amino acids that were also identified as being involved in DNA binding by crystal structure analysis (C) [29] or by NMR analysis (N) [30].

SDS-PAGE and subjected to trypsinolysis, and the resulting tryptic digests were analyzed by MALDI-TOF MS as well as by nanoLC/MALDI-TOF MS. The analysis was performed in reflector mode with detection in the 600 to $4000 \mathrm{~m} / \mathrm{z}$ range optimal for the detection of tryptic peptides. The peptide, which is crosslinked to the 21 mer DNA (molecular weight $\sim 8 \mathrm{kDa}$ ), is not detectable under these analytical conditions. Instead, it is identified by the absence of its signal in the peptide profile of the crosslinked protein.

The peptides detected by MALDI-TOF MS of the unseparated tryptic digests of the crosslinked and noncrosslinked protein were the same; therefore, the location of the crosslink could not be derived from the spectra. The relatively low sequence coverage of $56 \%$ (Fig. 4) can be attributed to signal suppression caused by the presence of two strong signals corresponding to the following DnaA tryptic peptides: ELTNHSLPEIGDAFGGR (aa 416-432, $\mathrm{m} / \mathrm{z}$ 1812.89) and EESHDIKEDFSNLIR (aa 449-463, $\mathrm{m} / \mathrm{z}$ 1831.88). The application of the recently established offline nanoLC/MALDI-TOF MS technique [26], which involves a separation step prior to MS analysis, permitted detection of peptides not observed with direct MALDI-TOF MS analysis of the peptide mixture. Consequently, combining both MS approaches led to nearly complete sequence coverage in the protein control (Fig. 4). The assignment of all DnaA tryptic peptides was confirmed by MS/MS analysis (data not shown).

To determine the DNA-binding site of the DnaA protein, nanoLC/MALDI MS analysis was performed on both the crosslinked protein-DNA and the noncrosslinked protein. The peptides detected in both samples were compared with respect to their $\mathrm{m} / \mathrm{z}$ values and retention times. Peptides with relative mass differences of less than $50 \mathrm{ppm}$ and retention time differences of less than $1 \mathrm{~min}$, corresponding to three LC/MALDI fractions, were considered to be identical. Fig. 5 shows the mass spectra acquired from three consecutive fractions for both samples. In these fractions, the same peptides are detected in the two samples with the exception of the peptide of $\mathrm{m} / \mathrm{z} 1152.6$ (indicated in boldface in Fig. 5A), which was detected only in the noncrosslinked protein. This was the only detected difference between the two data sets. MS/MS analysis of this compound (Fig. 6) and a fragment ion fingerprint search, using the MASCOT software (Matrixscience, UK), identified it as the peptide DHTTVLHACR (aa 433-442 of DnaA, Fig. 4). The absence of this peptide in the sample of the crosslinked protein-DNA (Fig. 5B) identifies it as the crosslinked peptide.

\section{Discussion}

In the current study, for the first time, we applied the protein microarray technology using a solid support in combination with fluorescence detection to analyze protein-DNA interactions. Ultraviolet crosslinking combined with MS located the site of the determined interaction. The studies were performed using the interaction of Escherichia coli DnaA with its cognate-binding site, the DnaA box, as a model. This interaction has been well characterized previously by gel retardation experiments [18], Biacore experiments [6], a solid-phase DNAbinding assay [22], crystal structure analysis [29], and nuclear magnetic resonance (NMR) analysis [30].

We used the DNA-binding domain 4 instead of the entire protein to overcome effects due to the homooligomerization of the protein and to restrict the analysis to undisturbed protein-DNA binding. The specific affinity of DnaA domain 4 for the DnaA box R4 is 

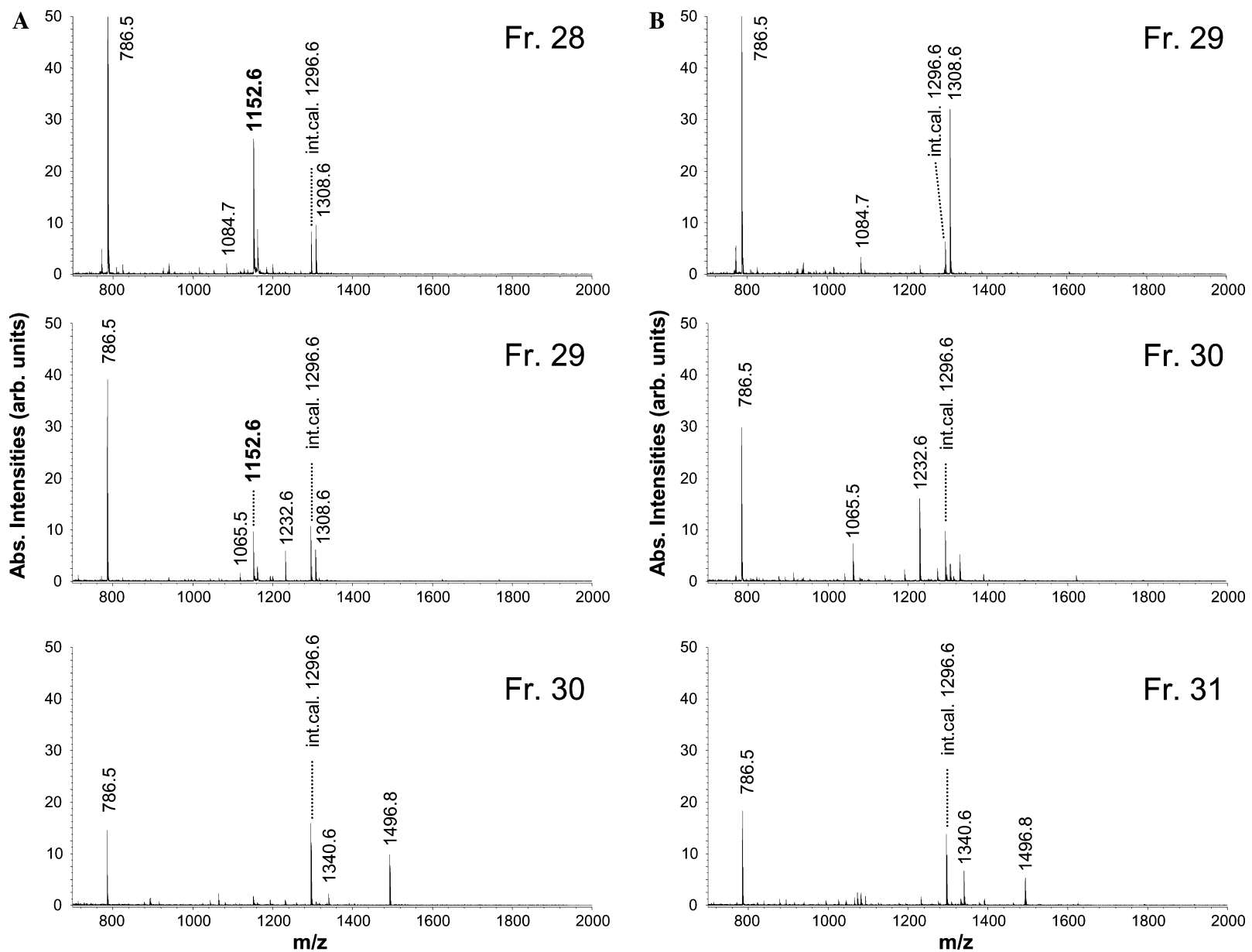

Fig. 5. MALDI-TOF mass spectra of three consecutive fractions of nano-LC separations of tryptic digests of (A) noncrosslinked protein and (B) crosslinked protein-DNA. The peptide corresponding to the signal at $\mathrm{m} / \mathrm{z} 1152.6$ (shown in boldface) coelutes with the peptides at $\mathrm{m} / z 1308.6$ and 1232.6 in the noncrosslinked protein sample. These reference signals are also present in the crosslinked protein-DNA sample, but no signal at $m / z$ 1152.6 was observed. The signal at $\mathrm{m} / z 1152.6$ was the only detected difference between the two samples, identifying the site of the DNA-protein crosslink.

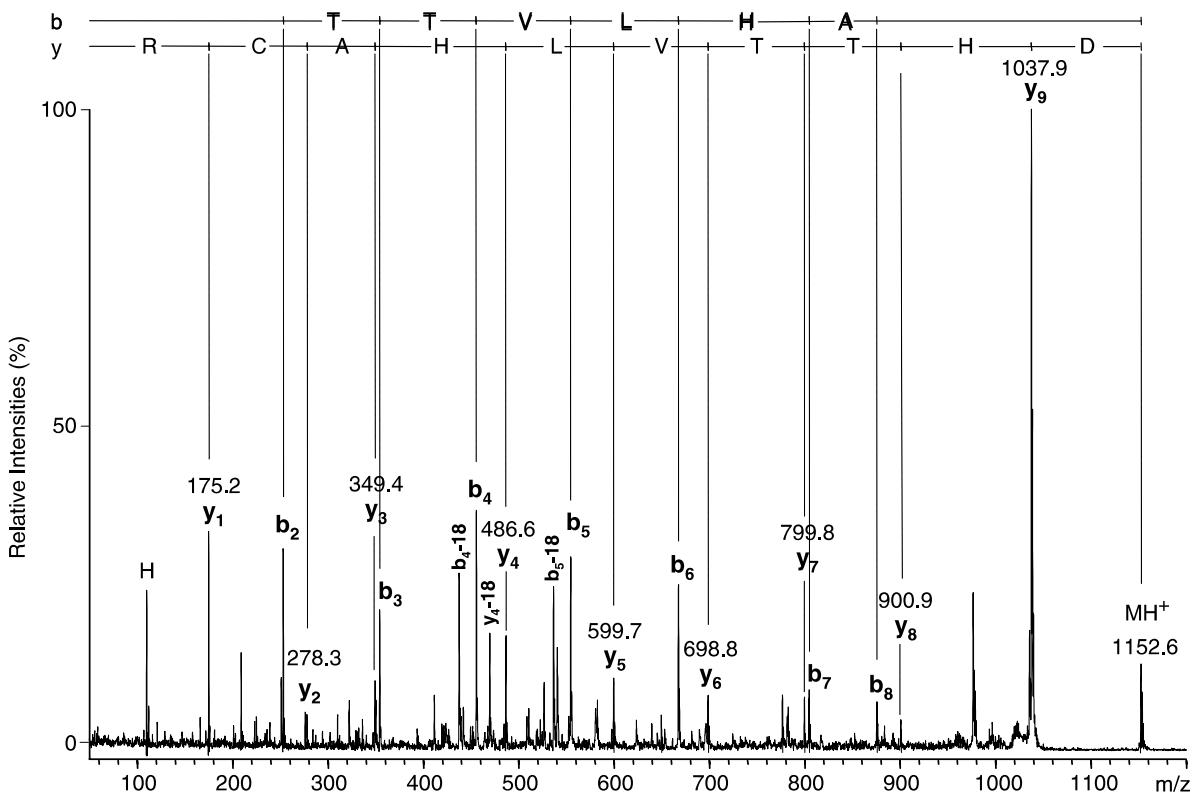

Fig. 6. MALDI-TOF MS/MS analysis of the peptide of $\mathrm{m} / \mathrm{z}$ 1152.6. A fragment ion fingerprint database search led to the identification of the peptide DHTTVLHACR, the assigned fragments of which are indicated in the figure. 
comparable to the affinity of the full-length DnaA as demonstrated by gel retardation experiments in this study and by Biacore experiments $\left(0.9 \mathrm{nM} K_{\mathrm{d}}\right.$ for fulllength DnaA [6]).

Previously, several other applications of protein microarrays have been described, for example, studies of molecular interactions of spotted proteins with antibodies, other proteins, small molecules, and lipids as well as the analysis of enzymatic reactions such as protein phosphorylation [9]. We used FAST slides that were used successfully previously to immobilize proteins or antibodies in other studies [31-33]. These slides are coated with a nitrocellulose-derived polymer, allowing a noncovalent attachment of the proteins that should allow the proteins to retain their native form. Using this protein microarray assay, we were able to monitor the interaction of $w t$ DnaA domain 4 with a dsOligo containing DnaA box R4 (Figs. 2 and 3), indicating that the native structure of the protein was retained after immobilization on the surface. The signal intensity increased with rising dsOligo concentations (2-2000 nM) (Fig. 3). A clear signal was still detectable after incubation of immobilized DnaA ( $24 \mathrm{fmol} / \mathrm{spot}$ ) with $50 \mathrm{nM}$ dsOligo. Lower protein concentrations were not tested. A direct comparison of this sensitivity with the recently published data of Snapyan and colleagues [11] obtained by studying protein-DNA interactions with membrane protein arrays is not possible because those authors studied interactions of other orders and used another detection principle.

To study the reliability of the protein microarray, we included a nonbinding mutant, the A440V DnaA domain 4 [6], in the experiments. The results obtained for the immobilized A440V DnaA domain 4 after incubation with DnaA box R4 dsOligo (Fig. 3) are in agreement with literature data for this mutant [6] and demonstrate the ability of the protein microarray technology to measure effects of mutations on the interaction specificity. Also, variations in the binding specificity due to changes in the DNA substrate used are detectable, as demonstrated by comparing the binding of $w t$ DnaA domain 4 to DnaA box 3 with that of $w t$ DnaA domain 4 to DnaA box R4 (Fig. 3). In contrast to the specific binding of DnaA box R4 to DnaA domain 4, no specific binding of DnaA box R3 was detectable. Only at the highest DnaA box R3 dsOligo concentration were unspecific signals obtained for $w t$ and mutant DnaA domain 4 as well as for the control protein (Fig. 3). These results are in agreement with literature data on DnaA box R3 [18,34,35].

The interaction of DnaA domain 4 with DnaA box R4 detected by protein microarray technology was further investigated by uv crosslinking combined with mass spectrometric analysis. As a first step, MALDI-TOF MS peptide mass fingerprinting of the crosslinked and noncrosslinked protein was performed. Because of MALDI signal suppression, which hampers the detection of some peptides in mixtures, the sequence coverage was insufficient for determination of the DNA attachment site (Fig. 4). For unambiguous identification of the crosslinked peptide, the analysis was optimized for high sequence coverage by the introduction of a chromatographic separation step prior to MS analysis. The analysis by nanoLC/MALDI-TOF MS led to nearly complete sequence coverage in the control protein and revealed a peptide missing only in the tryptic digest of the crosslinked protein (Figs. 4 and 5). This missing peptide, DHTTVLHACR, corresponds to the part of the protein sequence where the crosslink occurred (aa 433-442 of $E$. coli DnaA, Fig. 4). This result is in agreement with recently published structural data $[29,30]$. The exact location of the crosslink within the tryptic peptide was not further investigated, although the feasibility of doing this has been demonstrated with ESI MS [36,37].

The peptide fragment (aa 433-442) missing in the nanoLC/MALDI-TOF analysis (Fig. 5) should be involved in the specific protein-DNA contact. The 10 amino acid-enclosing fragment contains six residues (D433, H434, T435, T436, H439, and R442) that were also identified as being involved in DNA binding by crystal structure analysis [29] and by NMR analysis [30] of $E$. coli DnaA domain 4 (Fig. 4). All six of these amino acids recognize bases in the major groove of the DnaA box sequence. Amino acid exchanges at the histidine- 434 and threonine-435 positions led to severely reduced binding activities of the DNA-binding domain to DnaA box R4, as demonstrated by an extensive in vitro study of the DNA-binding domain [6].

Histidine-439, histidine-434, and threonine-435 recognize the thymidine T4 (T4 in the consensus sequence $5^{\prime}$ T-T-A/T-T-N-C-A-C-A). This base was substituted by bromodeoxyuracil in the crosslinking experiments performed in this study. 5-Bromodeoxyuracil closely resembles thymine structurally and can replace it in vivo [38]. The substitution of thymine residues by 5-bromodeoxyuracil promotes crosslinking of protein-DNA complexes on uv irradiation if the interaction partners are in close proximity (i.e., zero-length crosslinks). Aromatic amino acids (Tyr and Trp) are preferred residues for this reaction, but His, Cys, Lys, and Ala have also been reported to crosslink with 5-bromodeoxyuracil [39]. Therefore, histidine- 439 and histidine- 434 are good candidates for the amino acid being crosslinked to the target sequence.

Histidine-439 is located adjacent to alanine-440 that was mutated to valin in the used control nonbinding mutant A440V. This mutant leads to a nonbinding phenotype, as shown by in vivo and in vitro experiments [6]. We could verify this phenotype by gel retardation experiments and by protein microarray experiments. The NMR structure of $E$. coli DnaA domain 4 shows that $\mathrm{A} 440 \mathrm{~V}$ is located in the middle of an $\alpha$-helix involved in specific protein-DNA contact [30]. The exchange of 
alanine to valine may alter the structure of the involved $\alpha$-helix and result in the observed phenotype.

In the future, the technologies applied in this study will become important tools for studying protein-DNA interactions in high throughput, especially of transcription factors. In addition to dsDNA microarrays and the ChIP-chip technology, the application of protein microarray technology will help to reveal molecular targets of transcription factors. It enables the simultaneous analysis of numerous DNA-binding proteins in parallel experiments with respect to many DNA probes in small volumes. To reveal multi-input motifs in transcriptional regulatory networks [2], which are sets of regulators (transcription factors) that bind to a set of gene promoters, the application of protein microarrays may be very interesting because it allows the parallel selection of a set of transcription factors that recognize a given target DNA site in one hybridization. The inclusion of mutants of the protein into the microarray analysis may help to encircle amino acids or protein domains, which are important for the specific protein-DNA contact. Ultraviolet crosslinking of the protein with the DNA substrate in combination with nanoLC/MALDI-TOF MS can then be used as a rapid method for localization of protein regions involved in protein-DNA interactions.

\section{Acknowledgments}

We thank Margret Krause for cloning, expression, and purification of the proteins. We also thank Claus Hultschig for his encouragement and a lot of helpful discussions. We are grateful to Jasmin Bastian and Tanja Feilner for reading the manuscript.

This work was funded by the German Ministry for Research (BMBF Grants 031U102D, 031U202D, and 0312274) and the Max Planck Society.

\section{References}

[1] C.E. Samitt, F.G. Hansen, J.F. Miller, M. Schaechter, In vivo studies of DnaA binding to the origin of replication of Escherichia coli, EMBO J. 8 (1989) 989-993.

[2] T.I. Lee, N.J. Rinaldi, F. Robert, D.T. Odom, Z. Bar-Joseph, G.K. Gerber, N.M. Hannett, C.T. Harbison, C.M. Thompson, I. Simon, J. Zeitlinger, E.G. Jennings, H.L. Murray, D.B. Gordon, B. Ren, J.J. Wyrick, J.B. Tagne, T.L. Volkert, E. Fraenkel, D.K. Gifford, R.A. Young, Transcriptional regulatory networks in Saccharomyces cerevisiae, Science 298 (2002) 799-804.

[3] F. Jiang, S. Wisen, M. Widersten, B. Bergman, B. Mannervik, Examination of the transcription factor NtcA-binding motif by in vitro selection of DNA sequences from a random library, J. Mol. Biol. 301 (2000) 783-793.

[4] M.L. Bulyk, E. Gentalen, D.J. Lockhart, G.M. Church, Quantifying DNA-protein interactions by double-stranded DNA arrays, Nat. Biotechnol. 17 (1999) 573-577.

[5] C. Cicchini, H. Ansuini, L. Amicone, T. Alonzi, A. Nicosia, R. Cortese, M. Tripodi, A. Luzzago, Searching for DNA-protein interactions by lambda phage display, J. Mol. Biol. 322 (2002) 697-706.

[6] F. Blaesing, C. Weigel, M. Welzeck, W. Messer, Analysis of the DNA-binding domain of Escherichia coli DnaA protein, Mol. Microbiol. 36 (2000) 557-569.

[7] B. Kersten, B. Niemann, S. Jahn, Development of single-chain Fv fragments from a human anti-double-stranded DNA antibody to study the influence of somatic mutations on antigen binding, Exp. Clin. Immunogenet. 18 (2001) 96-99.

[8] J. Glökler, P. Angenendt, Protein and antibody microarray technology, J. Chromatogr. B Analyt. Technol. Biomed. Life Sci. 797 (2003) 229-240.

[9] H. Zhu, M. Snyder, Protein chip technology, Curr. Opin. Chem. Biol. 7 (2003) 55-63.

[10] H. Ge, UPA, a universal protein array system for quantitative detection of protein-protein, protein-DNA, protein-RNA, and protein-ligand interactions, Nucleic Acids Res. 28 (2000) e3.

[11] M. Snapyan, M. Lecocq, L. Guevel, M.C. Arnaud, A. Ghochikyan, V. Sakanyan, Dissecting DNA-protein and protein-protein interactions involved in bacterial transcriptional regulation by a sensitive protein array method combining a near-infrared fluorescence detection, Proteomics 3 (2003) 647-657.

[12] W. Messer, C. Weigel, Escherichia coli and Salmonella, Cellular, and Molecular Biology, ASM Press, Washington, DC, 1996.

[13] R.S. Fuller, B.E. Funnell, A. Kornberg, The DnaA protein complex with the E. coli chromosomal replication origin (oriC) and other DNA sites, Cell 38 (1984) 889-900.

[14] W. Messer, C. Weigel, DnaA initiator: also a transcription factor, Mol. Microbiol. 24 (1997) 1-6.

[15] W. Messer, C. Weigel, DnaA as a transcription regulator, Meth. Enzymol. 370 (2003) 338-349.

[16] H. Yoshikawa, N. Ogasawara, Structure and function of DnaA and the DnaA-box in eubacteria: Evolutionary relationships of bacterial replication origins, Mol. Microbiol. 5 (1991) 25892597.

[17] C. Schaefer, W. Messer, DnaA protein/DNA interaction: modulation of the recognition sequence, Mol. Gen. Genet. 226 (1991) 34 40.

[18] S. Schaper, W. Messer, Interaction of the initiator protein DnaA of Escherichia coli with its DNA target, J. Biol. Chem. 270 (1995) 17622-17626.

[19] C. Speck, C. Weigel, W. Messer, From footprint to toeprint: a close-up of the DnaA box, the binding site for the bacterial initiator protein DnaA, Nucleic Acids Res. 25 (1997) 3242-3247.

[20] M.Q. Fujita, H. Yoshikawa, N. Ogasawara, Structure of the DnaA and DnaA-box region in the Mycoplasma capricolum chromosome: conservation and variations in the course of evolution, Gene 110 (1992) 17-23.

[21] W. Messer, F. Blaesing, J. Majka, J. Nardmann, S. Schaper, A. Schmidt, H. Seitz, C. Speck, D. Tungler, G. Wegrzyn, C. Weigel, M. Welzeck, J. Zakrzewska-Czerwinska, Functional domains of DnaA proteins, Biochimie 81 (1999) 819-825.

[22] A. Roth, W. Messer, The DNA binding domain of the initiator protein DnaA, EMBO J. 14 (1995) 2106-2111.

[23] K. Bussow, D. Cahill, W. Nietfeld, D. Bancroft, E. Scherzinger, H. Lehrach, G. Walter, A method for global protein expression and antibody screening on high-density filters of an arrayed cDNA library, Nucleic Acids Res. 26 (1998) 5007-5008.

[24] R.A. Young, R.W. Davis, Yeast RNA polymerase II genes: isolation with antibody probes, Science 222 (1983) 778-782.

[25] U. Brinkmann, R.E. Mattes, P. Buckel, High-level expression of recombinant genes in Escherichia coli is dependent on the availability of the DnaY gene product, Gene 85 (1989) 109-114.

[26] E. Mirgorodskaya, C. Braeuer, K. D. Kloeppel, H. Lehrach, J. Gobom, Interfacing nanoLC and MALDI-TOF MS for the analysis of complex peptide mixtures, in: 51st ASMS Conference on Mass Spectrometry and Allied Topics, Montreal, 2003. 
[27] J. Gobom, M. Schuerenberg, M. Mueller, D. Theiss, H. Lehrach, E. Nordhoff, Alpha-cyano-4-hydroxycinnamic acid affinity sample preparation: A protocol for MALDI-MS peptide analysis in proteomics, Anal. Chem. 73 (2001) 434-438.

[28] J. Gobom, M. Mueller, V. Egelhofer, D. Theiss, H. Lehrach, E. Nordhoff, A calibration method that simplifies and improves accurate determination of peptide molecular masses by MALDITOF MS, Anal. Chem. 74 (2002) 3915-3923.

[29] N. Fujikawa, H. Kurumizaka, O. Nureki, T. Terada, M. Shirouzu, T. Katayama, S. Yokoyama, Structural basis of replication origin recognition by the DnaA protein, Nucleic Acids Res. 31 (2003) $2077-2086$.

[30] Y. Yoshida, T. Obita, Y. Kokusho, T. Ohmura, T. Katayama, T. Ueda, T. Imoto, Identification of the region in Escherichia coli DnaA protein required for specific recognition of the DnaA box, Cell. Mol. Life Sci. 60 (2003) 1998-2008.

[31] P. Angenendt, J. Glökler, J. Sobek, H. Lehrach, D.J. Cahill, Next generation of protein microarray support materials: evaluation for protein and antibody microarray applications, J. Chromatogr. A 1009 (2003) 97-104.

[32] B. Kersten, T. Feilner, A. Kramer, S. Wehrmeyer, A. Possling, I. Witt, M.I. Zanor, R. Stracke, A. Lueking, J. Kreutzberger, H. Lehrach, D.J. Cahill, Generation of Arabidopsis protein chips for antibody and serum screening, Plant Mol. Biol. 52 (2003) 9991010.

[33] T. Kukar, S. Eckenrode, Y. Gu, W. Lian, M. Megginson, J.X. She, $\mathrm{D}$. $\mathrm{Wu}$, Protein microarrays to detect protein-protein interactions using red and green fluorescent proteins, Anal. Biochem. 306 (2002) 50-54.

[34] M.R. Cassler, J.E. Grimwade, A.C. Leonard, Cell cycle-specific changes in nucleoprotein complexes at a chromosomal replication origin, EMBO J. 14 (1995) 5833-5841.

[35] C. Weigel, A. Schmidt, B. Ruckert, R. Lurz, W. Messer, DnaA protein binding to individual DnaA boxes in the Escherichia coli replication origin, oriC, EMBO J. 16 (1997) 6574-6583.

[36] H. Steen, J. Petersen, M. Mann, O.N. Jensen, Mass spectrometric analysis of a UV-cross-linked protein-DNA complex: Tryptophans 54 and 88 of E. coli SSB cross-link to DNA, Protein Sci. 10 (2001) 1989-2001.

[37] H. Steen, O.N. Jensen, Analysis of protein-nucleic acid interactions by photochemical cross-linking and mass spectrometry, Mass Spectr. Rev. 21 (2002) 163-182.

[38] F. Bonhoeffer, A. Gierer, J. Mol. Biol. 7 (1963) 534-540.

[39] K.M. Meisenheimer, T.H. Koch, Photocross-linking of nucleic acids to associated proteins, Crit. Rev. Biochem. Mol. Biol. 32 (1997) 101-140. 